\title{
Multibound solitons generation with a controllable number of bound states in a passive mode-locked all-fiber erbium-doped ring laser
}

Dmitriy A. Dvoretskiy, Stanislav G. Sazonkin, Igor S. Kudelin, llya O. Orekhov, Alexey B. Pnev, et al.

Dmitriy A. Dvoretskiy, Stanislav G. Sazonkin, Igor S. Kudelin, Ilya O. Orekhov, Alexey B. Pnev, Valeriy E. Karasik, Lev K. Denisov, "Multibound solitons generation with a controllable number of bound states in a passive modelocked all-fiber erbium-doped ring laser ," Proc. SPIE 11026, Nonlinear Optics and Applications XI, 110260Q (30 April 2019); doi: 10.1117/12.2520745

SPIE. Event: SPIE Optics + Optoelectronics, 2019, Prague, Czech Republic 


\title{
Multibound solitons generation with a controllable number of bound states in a passive mode-locked all-fiber erbium-doped ring laser
}

\author{
Dmitriy A. Dvoretskiy*a, Stanislav G. Sazonkin ${ }^{\mathrm{a}}$, Igor S. Kudelin ${ }^{\mathrm{b}}$, Ilya O. Orekhov ${ }^{\mathrm{a}}$, \\ Alexey B. Pnev ${ }^{a}$, Valeriy E. Karasik ${ }^{\mathrm{a}}$, Lev K. Denisov ${ }^{\mathrm{a}}$ \\ ${ }^{a}$ Bauman Moscow State Technical University, 2nd Baumanskaya str. 5-1, Moscow, Russia 107005 \\ ${ }^{\mathrm{b}}$ Aston Institute of Photonics Technologies Aston University, Birmingham, UK B4 7ET
}

\begin{abstract}
We have studied generation of stable and low-noise de-chirped ultrashort solitons in bound states and we have experimentally demonstrated the formation multi-bound solitons with the controllable number of bound states $7<N<17$ by pump power variation. A numerical simulation of the influence of various types of fluctuations on the generation mode was also carried out.
\end{abstract}

Keywords: optical frequency standard, optical frequency divider, erbium-doped fiber lasers, ultrashort pulse generation, femtosecond.

\section{INTRODUCTION}

Mode-locked (ML) ultrashort pulse (USP) fiber lasers can be treated as an ideal platform to expand future applications due to a complex nonlinear dynamics with a presence of a high value of a group velocity dispersion (GVD) and a third order dispersion (TOD) in the laser resonator [1]. Up to now a series of novel ML regimes have been investigated e.g. self-similar pulses [2, 3], noise-like pulses [4], multi-bound solitons [5] and a soliton rain generation [6]. Note that the overwhelming majority of these fiber lasers schemes are based on a passive ML by nonlinear polarization evolution (NPE) effect (or Kerr-effect) in optical fibers along [7] or by hybrid ML [8] i.e. in a co-action with saturable absorber such as single-walled carbon nanotubes [9, 10, 11], graphene [12] or SESAM [13].

Multi-bound solitons (MBS) generation regime, also known as soliton molecules, is of a considerable interest in various fields of applications, such as telecommunication transmission systems, in metrology field of optical frequencies, and etc. For example, the investigation of a MBS generation is a very attractive for increasing the data transfer capacity in telecommunications due to coding alphabet extension. The coding concept of MBS suggests a data stream using more than two symbols $\left(2^{\wedge} \mathrm{N}\right.$ symbols, where $\mathrm{N}$ is the number of generated solitons in a bound state) [1]. MBS have implementation in nonlinear photonic signal processing due to four-wave mixing process in nonlinear waveguides [14]. Moreover, MBS generation can be also used in a coherent pulse staking amplification scheme increasing an amplification efficiency along with a formation of high-energy solitons at a high-repetition rate [15].

Since the first numerical demonstration in Ref. [16] MBS generation have been obtained by different ML technics. For example, the highest repetition rate up to $10 \mathrm{GHz}$ can be achieved by active ML of a fiber laser [17, 18]. Passive mode-locking technics include generation of MBS by all-kinds of saturable absorbers such as SESAM [19, 20], carbon nanotubes [1, 21], graphene [22, 23] and $\mathrm{MoS}_{2}$ saturable absorber [24]. MBS generation have been also observed in NPE-based fiber lasers but stability of generation regime was unsatisfactory [25-28]. Up to now stable MBS generation have been obtained only with several ultrashort pulses in bound state.

Here we demonstrate the formation of stable multi-bound solitons with the controllable number of bound states at the telecommunication spectral window obtained in NPE-based all-fiber erbium-doped ring laser with a highly-nonlinear resonator.

\section{EXPERIMENTAL SETUP}

The experimental setup of the ML erbium-doped fiber (EDF) ring laser with a highly-nonlinear resonator is shown in Figure 1. A commercially developed isolator-polarizer (ISO PM) was used as an USP filter for launching ML based on the nonlinear polarization evolution effect and also ensured unidirectional generation. To adjust ML regime two polarization controllers (PCs) located at the both ends of the ISO PM were included into the ring cavity.

We have used a pigtailed single mode laser diode operating at $980 \mathrm{~nm}$ with a maximum output power of $500 \mathrm{~mW}$ as the pump source for the erbium-doped fiber (EDF). An 80/20 fiber coupler was used to lead the laser radiation out from the cavity. The all-fiber ring cavity was formed by an $3.28 \mathrm{~m}$ active EDF with a low signal core absorption of $\sim 14 \mathrm{~dB} / \mathrm{m}$ at the pump wavelength and with a dispersion $\mathrm{D} \sim-17.4 \mathrm{ps} /(\mathrm{nm} \cdot \mathrm{km})$ at $1550 \mathrm{~nm}$, highly nonlinear germanosilicate fiber (HNLF with a concentration of germanium oxide in the core $\sim 50 \mathrm{~mol} . \%$ ) with a dispersion D $100 \mathrm{ps} /(\mathrm{nm} \cdot \mathrm{km}$ ) at $1550 \mathrm{~nm}$ and $\sim 11.37 \mathrm{~m} \mathrm{SMF-28} \mathrm{fiber} \mathrm{(Corning} \mathrm{Corp.)} \mathrm{with} \mathrm{dispersion}$

Nonlinear Optics and Applications XI, edited by Mario Bertolotti, Alexei M. Zheltikov, Proc. of SPIE

Vol. 11026, 110260Q · @ 2019 SPIE · CCC code: 0277-786X/19/\$18 · doi: 10.1117/12.2520745 
$\mathrm{D} \sim 17.4 \mathrm{ps} /(\mathrm{nm} \cdot \mathrm{km})$ at $1550 \mathrm{~nm}$ was used to reduce the total net-cavity GVD to slightly negative region. The HNLF production parameters was the same as in [29] and the measured core diameter is $\sim 2.5 \mu \mathrm{m}$. The calculated value of the nonlinear refractive index $\mathrm{n}_{2}$ is $3.63 \cdot 10^{-16} \mathrm{~cm}^{2} / \mathrm{W}$.

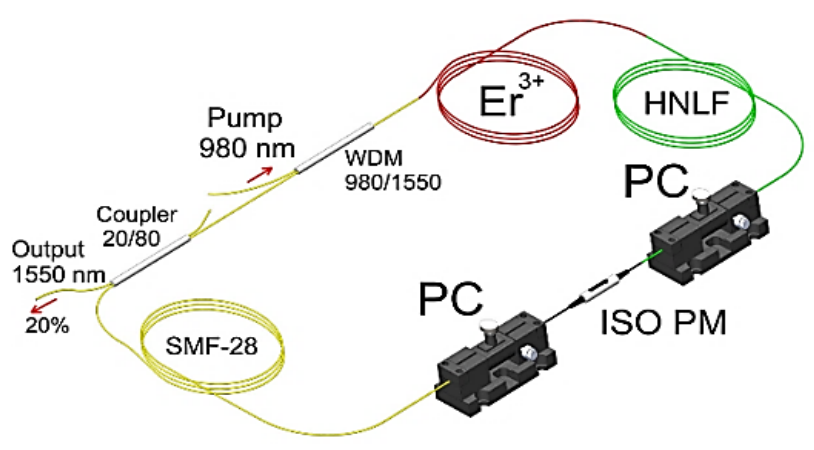

Figure 1. Experimental setup of the hybrid mode-locked erbium-doped all-fiber ring laser.

\section{EXPERIMENTAL RESULTS AND DISCUSSION}

Earlier we have achieved stretched pulses generation in the similar laser resonator with compensated net-cavity GVD and with a presence of significant third-order dispersion in the highly nonlinear laser resonator [30]. Here we demonstrate the formation of multiply coherently connected solitons occurring at a high pump power of $\sim 300 \mathrm{~mW}$. Output power versus pump power is shown on Figure 2(a) with a maximum average output power of $30 \mathrm{~mW}$. The MBS ML threshold was observed using proper PC settings with an average pump power of $\sim 160 \mathrm{~mW}$. Moreover, a hysteresis between threshold and decay of the regime against pump power was theoretically predicted for MBS generation [31].

a)

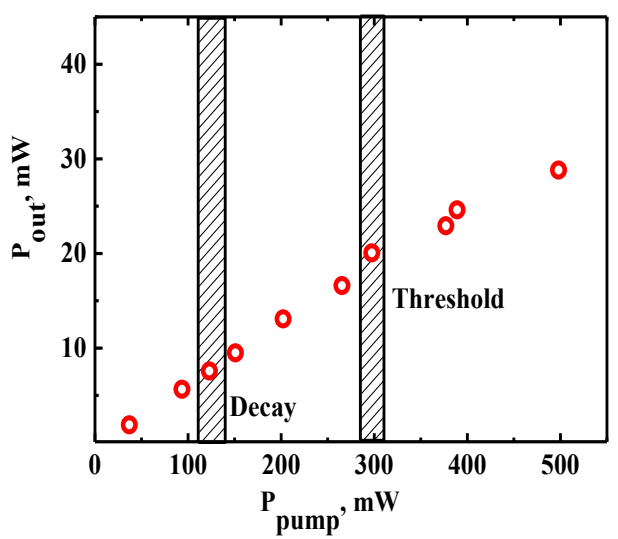

\section{b)}

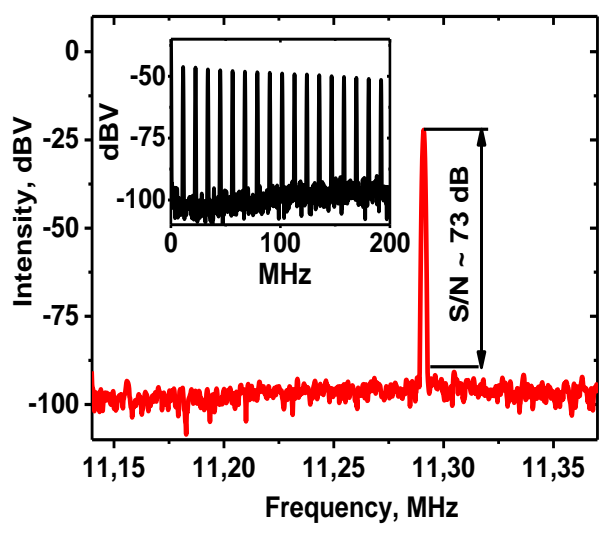

Figure 2.(a) Pump vs. output Power. (b) RF spectrum of the pulse train at the repetition frequency. Inset: RF spectrum in the range 0$200 \mathrm{MHz}$.

Figure 2(b) shows the typical RF spectrum for the MBS regime at the fundamental oscillator frequency with a resolution of $300 \mathrm{~Hz}$ (using the ESA FSL 3 model.03; Rohde \& Schwarz GmbH \& Co. KG, Munich, Germany). The RF spectrum has a peak at a fundamental frequency $\sim 11.3 \mathrm{MHz}$ with a signal-to-noise (SNR) ratio of $\sim 73 \mathrm{~dB}$, and the absence of any sidebands indicates the pulseto-pulse MBS energy stability. The inset of Figure 2(b) shows the RF spectrum in the frequency range $30 \mathrm{kHz}-200 \mathrm{MHz}(3 \mathrm{kHz}$ resolution bandwidth). The high SNR ratio at the fundamental frequency and the absence of any Q-switched sidebands in the RF spectrum prove the pulse-to-pulse stability of the ML regime. 


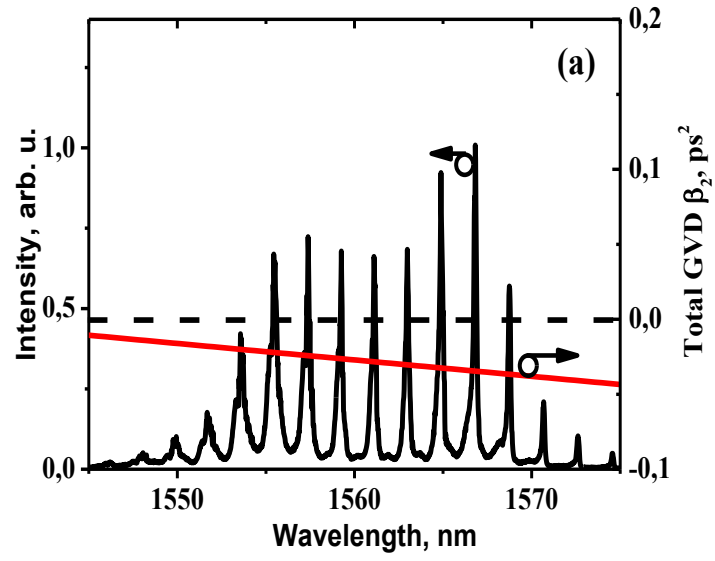

a)

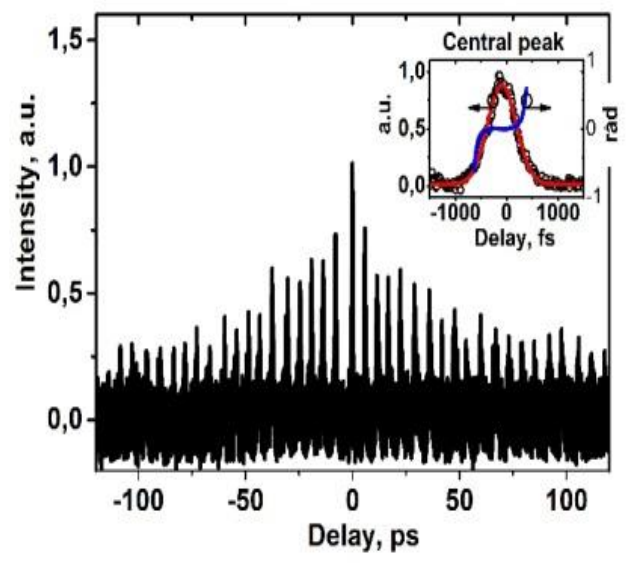

Figure 3. (a) Pulse spectra and net-cavity GVD. (b) Intensity autocorrelation trace. Inset: the central peak of the auto-correlation trace with Gaussian fitting and pulse phase.

The output spectrum (see Figure $3(\mathrm{a}))$ is evidently showing high-contrast intensity fringes $(\sim 13 \mathrm{~dB})$ that indicates a stable and strong coherent connection between ultrashort pulses and fixed coherent phase difference in the temporal domain between several identical pulses $[1,22,24]$. The lack of symmetry could be attributed as an high-order dispersion influence and the phase difference $\pi / 2$ between bound solitons [32]. Constant period of sharp intensity fringes indicates the presence of several bound pulses with stable time separation between them [27].

Figure 3 (b) shows the intensity autocorrelation trace of the observed MBS generation (obtained by using the autocorrelator FR103WS; FEMTOCHROME RESEARCH INC., Berkeley, CA, USA and USP phase by using Swamp Optics LLC, GRENOUILLE Model 15-40-USB, Georgia, USA) and central autocorrelation peak with Gaussian fitting and pulse phase (inset). More than 20 bound pulses generation is obtained with constant intertemporal width $\sim 4.5 \mathrm{ps}$ and with central peak duration of $<240 \mathrm{fs}$ at full width at half maximum (FWHM) assuming Gaussian fitting which is $\sim 19$ pulse widths between pulses. The period of the spectral modulation (see Fig. 4(a)) is $\Delta \lambda=2.5 \mathrm{~nm} \pm 0.5 \mathrm{~nm}(\Delta v=213.3 \mathrm{GHz})$. The spectral modulation inter-fringe is inversely proportional to the temporal separation of pulses $\tau=1 / \Delta v$. Thus, the calculated bound soliton separation $\tau=4.68 \mathrm{ps} \pm 1.2 \mathrm{ps}$ corresponds well to the experimental observation from the autocorrelation trace $(4.5 \mathrm{ps})$. The Gaussian form of the autocorrelation central peak without any pedestal and almost symmetrical form of autocorrelation without additional intensity peaks along with the observed output spectra indicates that one pulse duration in bond state $\tau_{\text {pulse }}<240 \mathrm{fs}$ at FHWM with stable temporal separation of pulses $\Delta \tau<\tau_{\text {pulse. }}$.

Our experimental results are confirmed by theoretical studies of the effect of the soliton energy quantization in a soliton all-fiber ring laser with passive mode locking based on the NPE effect in [3]. From which we concluded that the largest energy of a fundamental soliton with a duration $\tau_{\mathrm{p}}$ is limited by the soliton area theorem $\mathrm{E}_{\mathrm{s}} \sim\left|\beta_{2}\right| /\left(\gamma \bullet \tau_{\mathrm{p}}\right)$, where $\gamma$ is the total nonlinear coefficient, and $\beta_{2}$ is the total dispersion of the resonator. Quantization of the soliton energy leads to splitting of the pulse energy at operating powers exceeding the fundamental limit. Thus, the generation of MBS with $\mathrm{N} \sim 20$ bound states is a natural consequence of the amplification competition between multiple solitons and the generation of ultrashort short stretched pulses at high energies occurring in an all-fiber NPE-based erbium-doped laser with a highly nonlinear resonator [30].

However, in order to fully understand the nature of bound solitons formation, it was necessary to understand how laser parameters cause the appearance of a certain number of bound states, and, in addition, by changing these parameters, control the number of pulses in a bound state. To do this, the power of the pump source smoothly varied in the range from 160 $\mathrm{mW}$ to $\sim 377 \mathrm{~mW}$. It is worth noting that the generation type during the experiment have not changed, as can be clearly seen from the graphs of the spectra depending on the pump power (see Figure 4(a)), the main characteristics of the ML regime remained unchanged throughout the experiment.

Figure 4(b) shows the dependence of the intensity autocorrelation traces on the pump power (obtained using the autocorrelator FR103WS; FEMTOCHROME RESEARCH INC., Berkeley, California, USA). MBS generation with $7<\mathrm{N}<17$ bound pulses were obtained with a strongly fixed intertemporal width of $\sim 4.5 \mathrm{ps}$ for all pump powers and with a duration of the central peak of $<0.5 \mathrm{ps}$ at the full width at half maximum (FWHM). The Gaussian form of the autocorrelation central peak without any pedestal and the almost symmetric form of the autocorrelation trace without additional intensity peaks along with the observed output spectra indicate that the average pulse duration in the bound state is $\tau_{\text {pulse }}<0.5 \mathrm{ps}$ (at FWHM). 

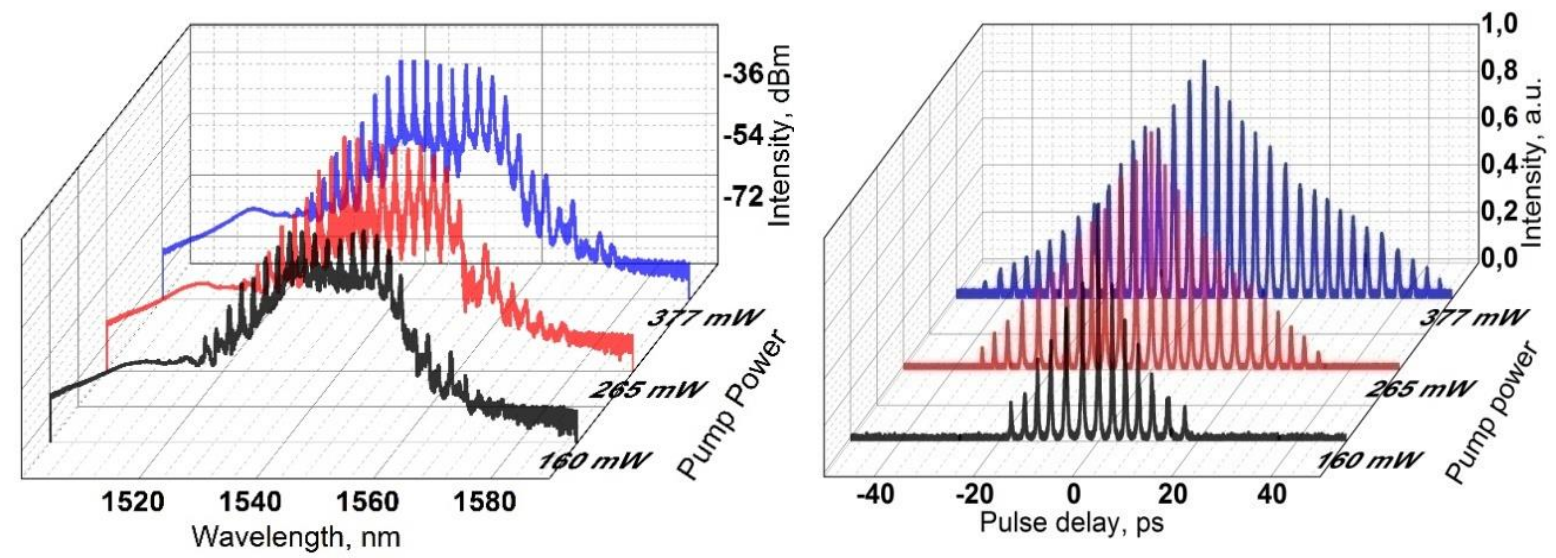

Figure 4. (a) The dependence of the output spectra on the pump power. (b) The dependence of the intensity autocorrelation trace on the pump power.

From soliton quantisation theory there are several way to vary the number of solitons. The simplest way is to change the pump power. As a result of our experiments, it was easy to determine the dependence of the number of bound states on the pump power. As it can be clearly seen from the autocorrelation traces, with increasing pump power the number of bound states increases. Thus, by varying the pump power, we can easily control the number of pulses in a bound state.

\section{NUMERICAL SIMULATIONS.}

In order to draw a conclusion about the stability and noises of the bound soliton generation we numerically investigated the influence of different kinds of fluctuation in one bound state (the temporal gap between neighbor solitons, pulse duration, amplitude and phase variations) on the optical spectrum and autocorrelation pattern. The simulations were provided for in-phase soliton bunch with $\mathrm{N}=20$, temporal separation of $4.5 \mathrm{ps}$ and pulse duration of $240 \mathrm{fs}$. The results of noise influence on output parameters are similar for solitons with $\mathrm{N}>4$ bound states.

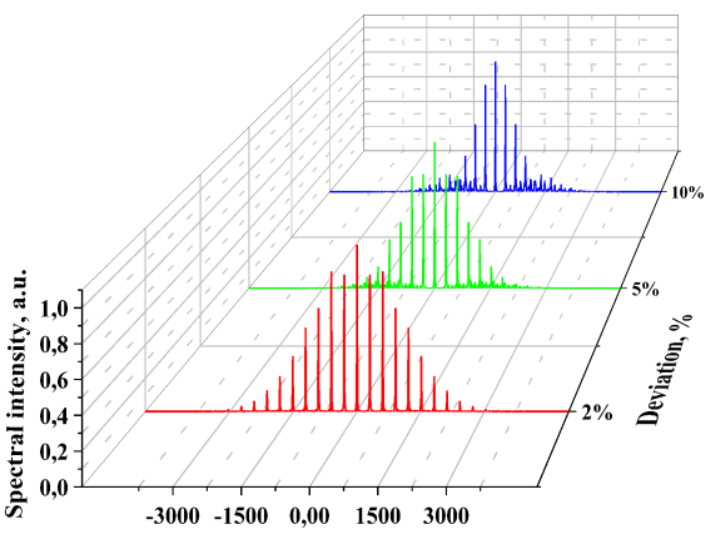

Normalized frequency, $\mathbf{G H z}$ a)

Figure 5. (a) Optical spectrum and (b) autocorrelation for 20 solitons in-phase $(\theta=0)$ in the presence of fluctuation of temporal separation between solitons in one bunch for the cases with maximum time deviation 2\%, 5\% and $10 \%$ (90 fs, $225 \mathrm{fs}$ and $450 \mathrm{fs}$ respectively) with standard deviation $\sigma=59 \mathrm{fs}, 144 \mathrm{fs}$ and $250 \mathrm{fs}$ respectively.

At first, we simulated fluctuations of the temporal separation $\Delta \mathrm{T}$ in one soliton molecule to see its influence on the optical spectrum and autocorrelation pattern. Figure 5(a) provides a simulated optical spectrum for $\mathrm{N}=20$ solitons in bound state in the presence of fluctuation of the temporal gap $\Delta \mathrm{T}$ between solitons with maximum time deviation of $2 \%, 5 \%$ and $10 \%$ (90 fs, $225 \mathrm{fs}$ and $450 \mathrm{fs}$ respectively) and standard deviation of $\sigma=59 \mathrm{fs}, 144 \mathrm{fs}$ and $250 \mathrm{fs}$ respectively. While the central peak is the same for all three cases, 
the modulation depth significantly decreases from the central peak to the edge and the peaks become less equidistant and noisier in the case of strong fluctuation of the time separation between solitons in bound state. Fig. 5 (b) depicts the intensity autocorrelation for these cases. While the central peak does not experience any changes, the edge peaks fall sharply in intensity and become significantly distorted in shape when the deviation of $\Delta \mathrm{T} \sim \tau_{\text {puse. }}$

At last we simulate various pulse duration $\mathrm{T}_{0}$ inside one soliton molecule and observe its optical spectrum and autocorrelation traces depicted on a Figure 6 . The pulse duration's deviations were $20 \%, 50 \%, 100 \%$ of $\mathrm{T}_{0}$ and standard deviation of $\sigma=18 \mathrm{fs}, 72 \mathrm{fs}$ and 104 fs respectively. It is clearly seen that optical spectrum become much noisier with lots of subpeaks and have new envelope modulations when pulse duration fluctuations become stronger. Therefore, the modulation depth of the optical spectrum provides general information on noises inside the soliton molecule. While the presence of well distinguishable edge peaks on spectrum signifies better quality of generated regime, the subpeaks give evidence about more significant fluctuations inside the molecule.

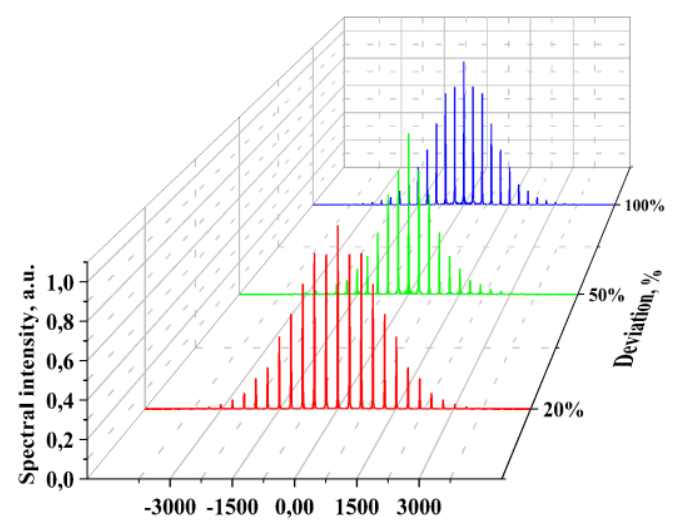

a)

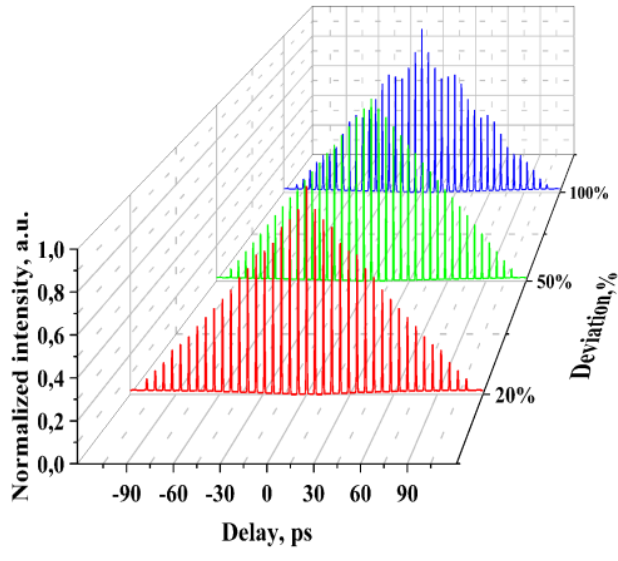

b)

Figure 6. (a) Optical spectrum and (b) intensity autocorrelation for 20 solitons in-phase $(\theta=0)$ with pulse duration fluctuation for the cases with maximum deviation $20 \%, 50 \%$ and $100 \%$ with standard deviation $\sigma=18 \mathrm{fs}, 72 \mathrm{fs}$ and 104 fs respectively.

Autocorrelation pattern has not experienced strong changes, but inaccuracies could be raised while obtaining pulse duration from the central peak. It is obviously that if there are several pulses in the bunch, the central peak of autocorrelation function presents a superposition of sum of each separated pulse with different width $\mathrm{T}_{0}$ and the central peaks cannot be approximated by Gaussian function with high accuracy (as it shown on Figure 7) when the pulse duration has strong fluctuation. It means, that the mismatch with Gaussian function can be treated as pulse duration fluctuations inside one molecule.

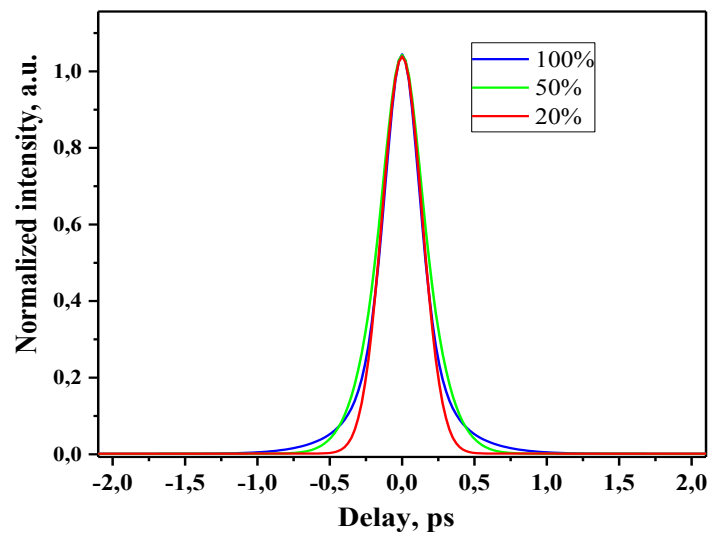

Figure 7. The central peak of the autocorrelation trace depicted on Figure 6(b).

In experiment, it takes time to shoot one autocorrelation pattern. This constitutes time of $\sim 300 \mathrm{~ms}$ to span $230 \mathrm{ps}$ (from the first to the last pulse in the soliton molecule) and almost $\sim 1 \mathrm{~ms}$ to span the central peak. Thus, a symmetry of the central peak of the autocorrelation 
trace denotes about short-term stability of the MBS generation (from $1 \mathrm{~ms}$ up to $300 \mathrm{~ms}$, or 11300 to 3390000 roundtrips). In addition, the central peak gives transitional pattern about the stability of the whole molecule.

\section{CONCLUSIONS.}

In summary, the generation of 20 stable and low-noise de-chirped ultrashort solitons in bound states with duration of $240 \mathrm{fs}$ at the repetition frequency of $\sim 11.3 \mathrm{MHz}$ (with a signal-to-noise ratio of $\sim 73.3 \mathrm{~dB}$ ) in the erbium-doped all-fiber ring laser with a highly-nonlinear resonator mode-locked by an NPE effect was observed. Also, we experimentally observe the formation of multi-bound solitons with the controllable number of bound states in the range of $7<\mathrm{N}<17$ by pump power variation. We have demonstrated the simplest method of pulse number controlling by changing the pump power. It should be noted that this method does not lead to the ML destruction in the pump power range from $\sim 160 \mathrm{~mW}$ to $\sim 377$ $\mathrm{mW}$. Moreover, the conducted numerical simulations confirm the generation of highly coherent MBS with high stability of the output characteristics.

The reported study was economically supported under Russian Foundation for Basic Research (RFBR) projects No. 1832-20017 and № 18-38-00615.

\section{REFERENCES.}

1. Chernysheva, M., et al., "Double-Wall Carbon Nanotube Hybrid Mode-Locker in Tm doped Fibre Laser: A Novel Mechanism for Robust Bound-State Solitons Generation,” Sci. Rep. 7, 44314 (2017)

2. Lazarev V., Krylov A., et al., "Stable similariton generation in an all-fiber hybrid mode-locked ring laser for frequency metrology," in IEEE Transactions on Ultrasonics, Ferroelectrics, and Frequency Control, vol.PP, no.99, pp. 1-1(2016).

3. I.O. Zolotovskii et al 2016 Quantum Electron. 461092

4. Mashiko Y., Fujita E., and Tokurakawa M., "Tunable noise-like pulse generation in mode-locked Tm fiber laser with a SESAM," Opt. Express 24, 26515-26520 (2016).

5. Nhan Duc Nguyen, Le Nguyen Binh, "Generation of high order multi-bound solitons and propagation in optical fibers," Optics Communications, Volume 282, Issue 12, 2394-2406 (2009)

6. Alioune Niang, Foued Amrani, , et al., "Rains of solitons in a figure-of-eight passively mode-locked fiber laser," Applied Physics B, Volume 116, Issue 3, pp 771-775 (2014).

7. K. Tamura, E. P. Ippen, H. A. Haus, and L. E. Nelson, "77-fs pulse generation from a stretched-pulse mode-locked all-fiber ring laser," Opt. Lett. 18, 1080-1082 (1993)

8. Xiang Zhang, Hongyu Hu, Wenbo Li, and Niloy K. Dutta, "High-repetition-rate ultrashort pulsed fiber ring laser using hybrid mode locking," Appl. Opt. 55, 7885-7891 (2016)

9. D. A. Dvoretskiy et al., "Comb Peculiarities of Dispersion-Managed Solitons in a Hybrid Mode-Locked All-Fiber Ring Laser," in IEEE Photonics Technology Letters, vol. 29, no. 18, pp. 1588-1591 (2017)

10. Chernysheva, M et al., "Carbon nanotubes for ultrafast fibre lasers," Nanophotonics, Volume 6, Issue 1, id.156, 30pp. (2017)

11. A.A. Krylov et al., "Ultra-short pulse generation in the hybridly mode-locked erbium-doped all-fiber ring laser with a distributed polarizer," Laser Physics Letters Volume 12, Issue 6, p. 06500 (2015).

12. Chen, K., et al., "All-polarization-maintaining fiber laser mode-locked by graphene,” Hongwai yu Jiguang Gongcheng/Infrared and Laser Engineering, 46 (10) (2017)

13. U. Keller, et al., "Semiconductor saturable absorber mirrors (SESAM's) for femtosecond to nanosecond pulse generation in solid-state lasers," IEEE Journal of Selected Topics in Quantum Electronics 2, pp. 435-453 (1996)

14. Optical Multi-Bound Solitons. Optics and Photonics by Le Nguyen Binh, Taylor \& Francis, 2015 ISBN 1482237636 , 9781482237634

15. Can Kerse, Hamit Kalaycıŏglu, Parviz Elahi, Barbaros Çetin, Denizhan K. Kesim, Önder Akçaalan, Seydi Yavaş, Mehmet D. Aşık, Bülent Öktem, Heinar Hoogland, Ronald Holzwarth and Fatih Ömer Ilday "Ablation-cooled material removal with ultrafast bursts of pulses," Nature volume 537, pages 84-88 (2016)

16. Tong Zhou, John Ruppe, et al. Opt. Express 23, 7442-7462 (2015)

17. Boris A. Malomed, "Bound solitons in coupled nonlinear Schrödinger equations," Phys. Rev. A 45, R8321 (1992)

18. C. Luo, S. Wang, and Y. Lai, "10 GHz Bound Soliton Mode-locking in an Environmentally Stable FM Mode-locked Erdoped Fiber Soliton Laser," in CLEO: 2014, OSA Technical Digest (online) (Optical Society of America, 2014), paper JTu4A.69.

19. Nhan Duc Nguyen, Le Nguyen Binh, Generation of high order multi-bound solitons and propagation in optical fibers, Optics Communications, Volume 282, Issue 12, 2009, Pages 2394-2406 
20. Zolotovskii,I O et al. Quantum Electronics(2015),45(1):26

21. Shuqian Sun, Zhixing Lin, Wei Li, Ninghua Zhu, and Ming Li, "Time-stretch probing of ultra-fast soliton dynamics related to Q-switched instabilities in mode-locked fiber laser," Opt. Express 26, 20888-20901 (2018)

22. H. H. Liu and K. K. Chow, "High Fundamental-Repetition-Rate Bound Solitons in Carbon Nanotube-Based Fiber Lasers," in IEEE Photonics Technology Letters, vol. 27, no. 8, pp. 867-870, 15 April15, 2015.

23. Y. F. Song, H. Zhang, L. M. Zhao, D. Y. Shen, and D. Y. Tang, "Coexistence and interaction of vector and bound vector solitons in a dispersion-managed fiber laser mode locked by graphene," Opt. Express 24, 1814-1822 (2016)

24. Jakub Bogusławski; Grzegorz Soboń; Iwona Pasternak; Aleksandra Krajewska; Włodek Strupiński; Krzysztof M. Abramski; Jarosław Sotor Bound soliton state in all-polarization maintaining fiber laser mode-locked by graphene Proceedings Volume 10159, Laser Technology 2016: Progress and Applications of Lasers; 1015907 (2016)

25. Yadong Wang, Dong Mao, Xuetao Gan, Lei Han, Chaojie Ma, Teli Xi, Yi Zhang, Wuyun Shang, Shijia Hua, and Jianlin Zhao, "Harmonic mode locking of bound-state solitons fiber laser based on MoS2 saturable absorber," Opt. Express 23, 205210 (2015)

26. Philippe Grelu, Franck Belhache, François Gutty, and Jose M. Soto-Crespo, "Relative phase locking of pulses in a passively mode-locked fiber laser," J. Opt. Soc. Am. B 20, 863-870 (2003)

27. Ph. Grelu, F. Belhache, F. Gutty, and J.-M. Soto-Crespo, "Phase-locked soliton pairs in a stretched-pulse fiber laser," Opt. Lett. 27, 966-968 (2002)

28. N. H. Seong and Dug Y. Kim, "Experimental observation of stable bound solitons in a figure-eight fiber laser," Opt. Lett. 27, 1321-1323 (2002)

29. Y. Yatsenko and A. Mavritsky, "D-scan measurement of nonlinear refractive index in fibers heavily doped with GeO2," Opt. Lett. . 32, 3257-3259 (2007)

30. Dmitriy A. Dvoretskiy, Stanislav G. Sazonkin, Ilya O. Orekhov, Igor S. Kudelin, Alexey B. Pnev, Valeriy E. Karasik, Alexandr A. Krylov, and Lev K. Denisov, "High-energy ultrashort-pulse all-fiber erbium-doped ring laser with improved freerunning performance," J. Opt. Soc. Am. B 35, 2010-2014 (2018)

31. Andrey Komarov, Herve Leblond, and François Sanchez "Multistability and hysteresis phenomena in passive mode-locked lasers", PHYSICAL REVIEW A, 71, 053809, 2005

32. Lili Gui , Pan Wang, Yihang Ding, Kangjun Zhao, Chengying Bao, Xiaosheng Xiao and Changxi Yang, "Soliton Molecules and Multisoliton States in Ultrafast Fibre Lasers: Intrinsic Complexes in Dissipative Systems", Applied Sciences 2018, 8(2), 201 